\title{
Assessment of Quarter Cylinder Obstacles Effectiveness on Hydraulic Jump in Rectangular Channels
}

\author{
Hassan Saghi i", Abdolamir Bak Khoshnevis² and Ali Saman² \\ 'Department of Civil Engineering, Hakim Sabzevari University, Iran; saeedi260@gmail.com \\ ${ }^{2}$ Department of Mechanical Engineering, Hakim Sabzevari University, Iran
}

\begin{abstract}
In this paper, the effect of quarter cylinder obstacles on hydraulic jump in a rectangular channel has been studied in the laboratory. The quarter-cylindrical obstacles with 2 and $3 \mathrm{~cm}$ diameter are considered and the effect of their position and height are studied in the hydraulic jump parameters such as location, energy dissipation and Froude number. The flume used in this research has six meter long, $20 \mathrm{~cm}$ wide and $30 \mathrm{~cm}$ height. The reason of quarter-cylindrical shape selection for obstacles is reduce the amount of erosion relative to other shapes such as rectangular and trapezoidal obstacles. The obstacles are installed at the end of the channel. Distance between the first obstacle and the beginning of the channel is constant. Totally, 44 tests have been carried out in this research. The results show that increasing distance between the obstacles to a certain amount, increases energy dissipation. But energy dissipation decreases for further distance. Furthermore, optimum $\mathrm{s} / \mathrm{r}$ increases with increase in the obstacles height.
\end{abstract}

Keywords: Energy Dissipation, Froude Number, Hydraulic Jump, Obstacle

\section{Introduction}

In order to prevent the damages caused by the huge extraordinaire energy of water in extra critical speeds and also in order to destroy extra kinetic energy existing in such currents, it is generally required to use special structures called energy dissemination that are made in flow downstream. In addition to destroying the energy of water, these kind of structures are considered as a suitable means for control and inhibitor of hydraulic jump and preparing the ground for its occurrence in a special location. In other words, when a hydraulic jump occurs on a horizontal smooth surface, any smallest change in the depth of upstream or downstream flows can change the jump location to either end. When the jump occurs in a special point and its oversensitivity against deep flow fluctuations is restricted, the structures known as hydraulic jump inhibitors have to be used. These structures are also used to determine the exact place of hydraulic jump in unroofed canals for restoring the bed in the place of jump and preventing extra charges for restoring the whole canal bed. A number of studies have been made by different researches on hydraulic jumps on rough bed.

For instance Pagliara et al. investigated energy drop on stone slopes with rather low slope by making models in different slopes (1:12 to 1:4) using stone materials in different sizes as khoshkeh chin. Their aim was to study the stones layout on the stability of torrent as well as the structure's energy dissipation'. Beirami and Hosseini studied the effect of distance between continued walls on the bed on controlling hydraulic jump. They showed that increase in the wall height has a reducing role of secondary depth and the length of whirlpool jump ${ }^{2}$. Shukry also made studies on hydraulic jump control with a continued wall. He showed that the distance between wall and jumping claw and the wall height influences the manner of forming the flow passing on the wall ${ }^{3}$. In ${ }^{4}$ it was investigated the effect of triangle barriers on the amount of energy dissipation. In that the amount of energy dissipation in hydraulic jump in a bed with triangle roughness

${ }^{*}$ Author for correspondence 
was 18 percent more than classic jump. Abbaspour et al. studied on wave triangular roughness and gained similar results ${ }^{5}$ In $^{6}$ the effect of cubic roughness on hydraulic jump and energy dissipater was studied. Because of creating cavitation in the bed roughness, wave beds were suggested ${ }^{7}$. Gohari and Farhoudi did their experiments within Froude numbers 3 to 10 . They saw that the secondary depth of jump on rough bed has a decrease as compared to channel with smooth bed and this reduction increases with the increase in the roughness's ${ }^{8}$.

In most studies, the barriers and roughness's fixed in the bed for controlling hydraulic jump that has been the cause of jump in the valve. The positions of these barriers in the place of jump and their effect on the characteristics of jump have been studied. Thus, the present article laboratory studies are made to understand the effect of dimensions and distances of quadrant barriers on the specifications of hydraulic jump including length, secondary height, the place of formation. The amount of energy dissipation is also studied and the findings have been presented and analyzed.

\section{The Description}

The tests were done in a rectangular channel $6 \mathrm{~m}$ long, $0.2 \mathrm{~m}$ wide and $0.3 \mathrm{~m}$ high in the laboratory of fluids in Hakim Sabzevari University in Sabzevar. The desired discharge was supplied by a centrifuge pump in which changes in discharge entering the canal is possible by the tap inserted before the canal entry ${ }^{1}$. Making changes in canal slope is also possible. A rectangular overflow in the entry of the canal is fixed to create hydraulic jump and the barriers are fixed at the end of it. The water gauge has been designed to measure the flow depth with $0.1 \mathrm{~mm}$ error and also it is calibrated with canal bed to make the work easy and the vector data has been done precisely. The gauge can to move parallel to the canal so that the depth can be measured in each point of the canal. In order to create hydraulic jump, the arched crown of overflow is fixed with an angle from the bottom. The valve at the end of the canal is completely open so that only the effects of the barriers can be studied. In order to calculate the discharge passing from a triangular overflow in primary reservoir according to the equation 1 (Figure 1) in which Q stands for passing discharge and $\mathrm{h}$ stands for water height inside the reservoir behind the triangular overflow.

$$
Q=1.35 h^{2.5}
$$

The schematic plan of the area being studied and the manner of installing barriers at the end of the canal to the flow direction is shown in Figure 2 and 3 respectively. All the used parameters in Figure 2 are shown in Table 1.

\section{Governing Equations}

The amount of energy existing in each point of the flow in an open canal with a slope less than 6 degree can be defined by Bernoulli equation as follows?

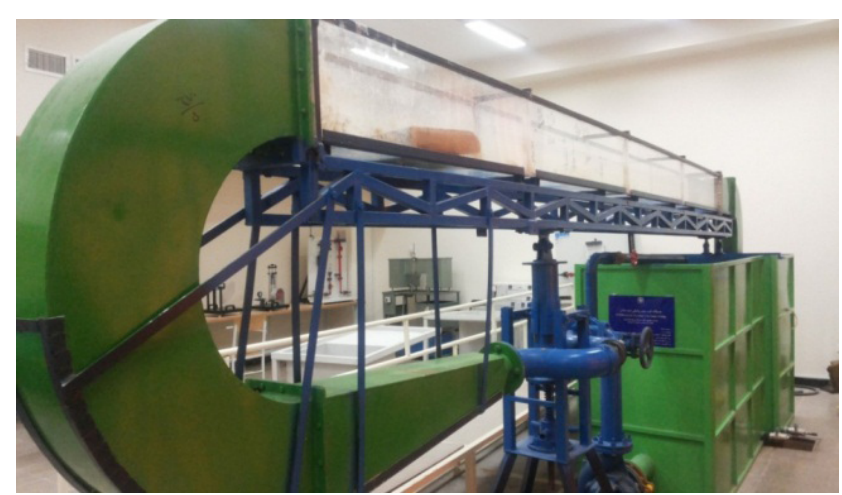

Figure 1. Flum used to study the effect of barriers on hydraulic jump.

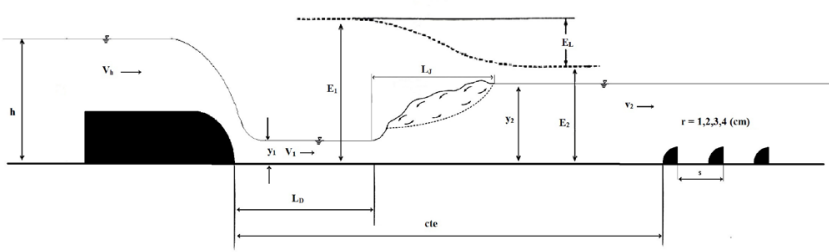

Figure 2. The schematic plan of peak overflow and the barriers installed in the canal.

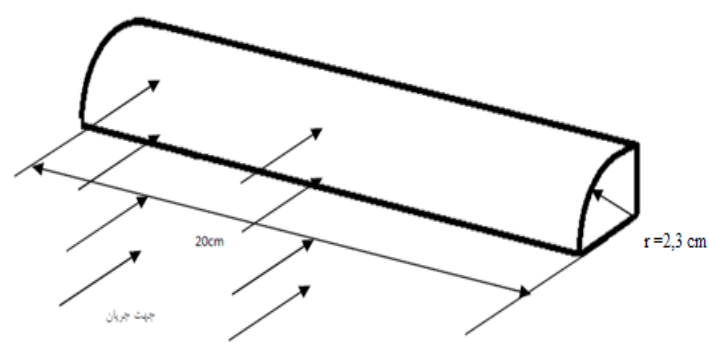

Figure 3. The direction of barriers installation toward the flow direction. 
Table 1. The parameters used in Figure 3.

\begin{tabular}{|c|c|}
\hline $\mathrm{H}$ & Depth of the rectangular overflow \\
\hline $\mathrm{y}_{1}$ & Depth of the super critical flow \\
\hline$y_{2}$ & Depth of the super critical flow \\
\hline $\mathrm{L}_{\mathrm{D}}$ & $\begin{array}{l}\text { distance of the beginning of the jump formation } \\
\text { from rectangular overflow claw }\end{array}$ \\
\hline $\mathrm{L}_{\mathrm{j}}$ & Length of hydraulic jump \\
\hline $\mathrm{z}$ & Depth of Triangular overflow \\
\hline $\mathrm{r}$ & Obstacle height \\
\hline S & Wavelength of the corrugation \\
\hline Q & Volumetric discharge \\
\hline $\mathrm{q}$ & Discharge per unit width of the flow \\
\hline $\mathrm{V}_{\mathrm{h}}$ & Average velocity before rectangular overflow \\
\hline $\mathrm{V}_{1}$ & Average velocity of supercritical flow \\
\hline $\mathrm{V}_{2}$ & Average velocity of subcritical flow \\
\hline $\mathrm{Fr}_{1}$ & initial Froude number \\
\hline $\mathrm{E}_{1}$ & specific energy before the jump \\
\hline $\mathrm{E}_{2}$ & specific energy after the jump \\
\hline $\mathrm{E}_{\mathrm{L}}$ & Energy loss \\
\hline $\mathrm{R}_{\mathrm{L}}$ & Relativity energy loss \\
\hline
\end{tabular}

$$
E=p / \gamma+z+\alpha v^{2} / 2 g
$$

In which $\mathrm{Z}$ is the distance of the desired point from the base level, $\mathrm{P}$ stands for pressure, $\mathrm{Y}$ is the specific gravity of the fluid, $\mathrm{V}$ is the flow speed, a stands for the of kinetic energy correction coefficient and $g$ stands for gravity acceleration. In this equation $\mathrm{p} / \mathrm{y}$ are called pressure head and $v^{2} / 2 g$ is called speed head. in case the canal bottom is considered as base level and as $p / y=y$ (y stands for water depth in canal bottom), the above equation is used as follows.

$$
E=y+\alpha v^{2} / 2 g
$$

Since the above equations have been written based on one dimensional analysis, the correction coefficient $\alpha$ has been considered as unit (1). Thus the above equation changes to the following one so-called specific energy ${ }^{10}$. The Figure 4 shows the diagram of changes in specific energy for a fixed discharge and for different depths.

Energy drops in the length of the jump $\left(\mathrm{E}_{\mathrm{L}}\right)$ and the relative energy drop in a hydraulic jump $\left(R_{L}\right)$ is gained through equations 4 and 5 respectively.

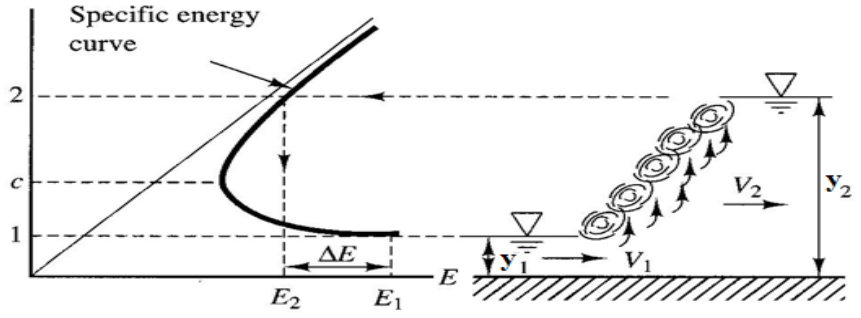

Figure 4. The diagram of changes of specific energy for a fixed discharge and for the depths.

$$
\begin{gathered}
E_{L}=\left(y_{2}+\alpha v_{2}^{2} / 2 g\right)-\left(y_{1}+\alpha v_{1}^{2} / 2 g\right) \\
R_{L}=\left(E_{L} / E_{1}\right) \times 100
\end{gathered}
$$

\section{Findings}

In this research, the effects of the barriers installed at the end of the canal on creating jump, the place jump is formed; the amount of energy dissipation and the changes of the primary Froude number were studied. For this purpose, 4 barriers with the height of 1 to $4 \mathrm{~cm}$ were placed in the way of the flow and its effect on the specifications of hydraulic jump specifications was assessed. In the conditions in which $1 \mathrm{~cm}$ barriers were used, hydraulic jump was not formed and in the conditions $4 \mathrm{~cm}$ barrier was used, the jump was submerged. Thus only the findings related to 2 and $3 \mathrm{~cm}$ are presented in this study. In this direction, in each test, the number of 3 barriers with a defined height at the end of each canal were placed in 3-m distance from rectangular overflow in the bottom of the canal in a way that the distance between the barriers can change. The barriers were installed on the bottom in a way that they can be easily detached and they do not hurt the bottom of the canal. Vector data were done in d different discharges. In all cases the place of installing the first barrier is fixed. Then the effect of barriers specifications on the flow Froude number, the point jump was formed and the energy dissipation was separately studied.

\subsection{The Effect of Barriers Specifications on the Froude Number of the Flow}

Considering the hydraulic jump categorization based on primary Froude, increase in Froude number in the primary phase of the hydraulic jump will lead to sustainable and fixed jump. Thus in order to study the effect of 
barriers on the type of hydraulic jump, barriers with the height of $\mathrm{r}=2,3 \mathrm{~cm}$ in different distances $\mathrm{s}$ were placed in the way of the flow and the changes in Froude number related to primary depth of hydraulic jump per different values of discharge and relative roughness $(\mathrm{s} / \mathrm{r})$ were measured as shown in Figures 5 and 6 respectively. The findings show that increase in discharge causes increase in the rate of primary Froude number. Also the maximum Froude number for $2 \mathrm{~cm}$ barriers in the range $(\mathrm{s} / \mathrm{r}=2,3)$ and for $3-\mathrm{cm}$ barriers in the range $(\mathrm{s} / \mathrm{r}=3,4)$. This indicates that the amount of optimal relative roughness is in direct relationship with increase in height of the barriers. The height of the quadrant barriers increases, the optimal relative roughness will also be more.

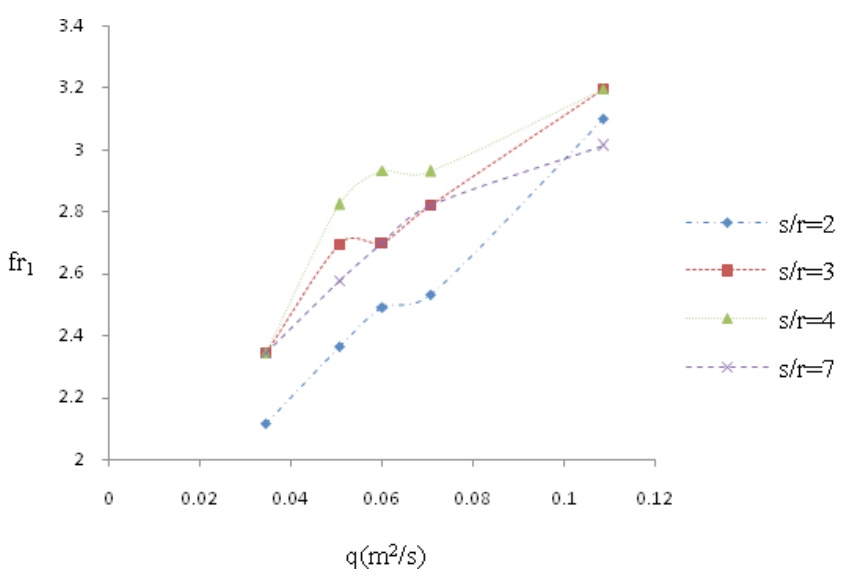

Figure 5. The effect of relative roughness on the changes of Froude number for $3 \mathrm{~cm}$ barriers.

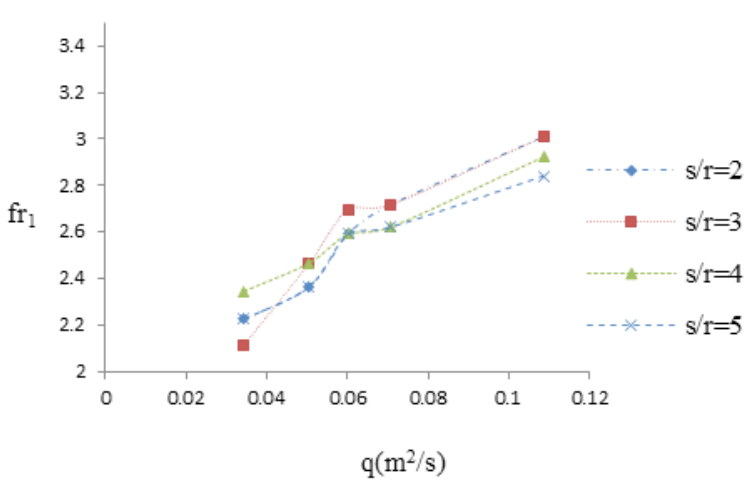

Figure 6. The effect of relative roughness on the changes of Froude number for $2 \mathrm{~cm}$ barriers.

\subsection{The Effect of Barriers Specifications on Energy Dissipation}

The goal of creating hydraulic jump in the way of this flow is to increase energy dissipation. Thus in this part, the percentage of relative energy dissipation per different values of discharge and relative roughness of the barriers is calculated and shown in Figures 7 and 8.

Figure 7 indicates that in a constant discharge, the most rate of energy dissipation is seen in relative roughness $(\mathrm{s} / \mathrm{r}=4)$. Similarly, the most amount of energy dissipation happens in maximum discharge. Moreover in a constant discharge, by increasing the distance between the barriers up to $12 \mathrm{~cm}$, the rate of energy dissipation increases that is an optimal point and after that by increasing the distance between the barriers for instance for relative roughness $(\mathrm{s} / \mathrm{r}=7)$, the amount of dissipation decreases. The findings gained in fig. 8 also shows that the most

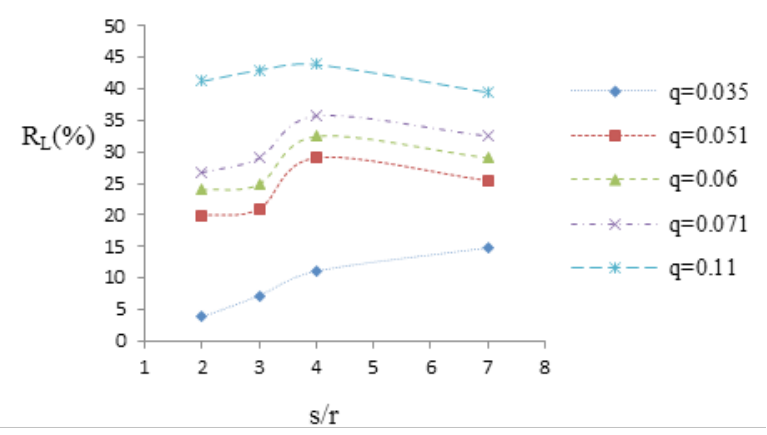

Figure 7. The effect of relative roughness factor on the amount of energy dissipation in different discharges $(3-\mathrm{cm}$ barriers).

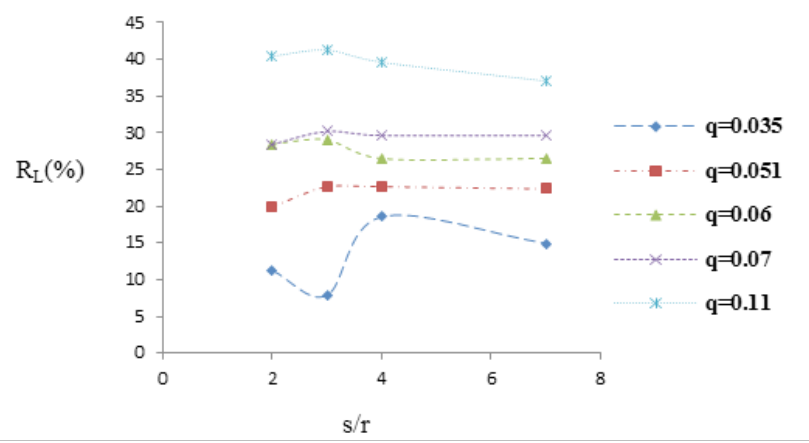

Figure 8. The effect of relative roughness factor on the amount of energy dissipation in different discharges (2-cm barriers). 
amount of dissipation in a constant discharge occurs in relative roughness $(\mathrm{s} / \mathrm{r}=3)$. By increase in the distance between the barriers the amount of dissipation decreases. In this stage, the Froude number changes is calculated based on the amount of energy dissipation based on relative roughness as shown in Figures 9 and 10.

The findings show that increase in Froude number cause increase in the amount of energy dissipation and this value has roughly been the same in $3-\mathrm{cm}$ barriers by changing the distance between the barriers. The most amount of dissipation has been seen in relative roughness 4 that is $43 \%$. The findings for 2 -cm barriers show that increase in Froude number causes increase in energy dissipation and the most energy dissipation in relative roughness 2 and 3. Optimal relative roughness increases with increase in height of quadrant barriers.

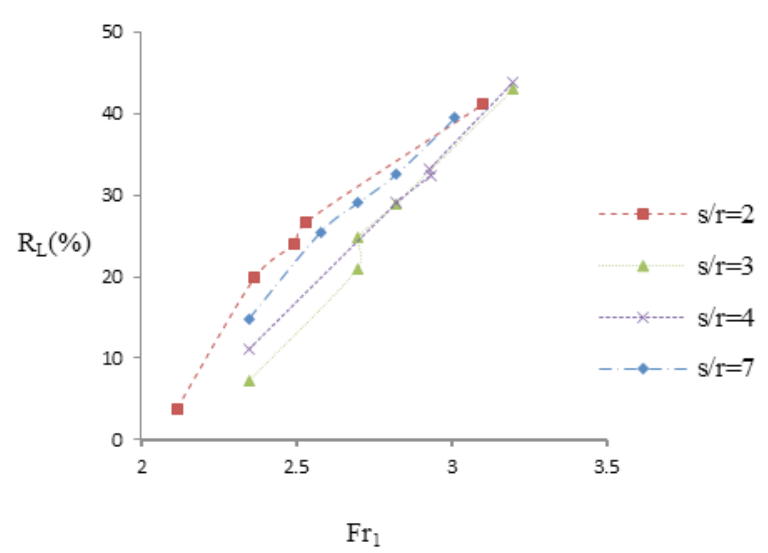

Figure 9. Energy dissipation changes in primary Froude number ( $3 \mathrm{~cm}$ barriers).

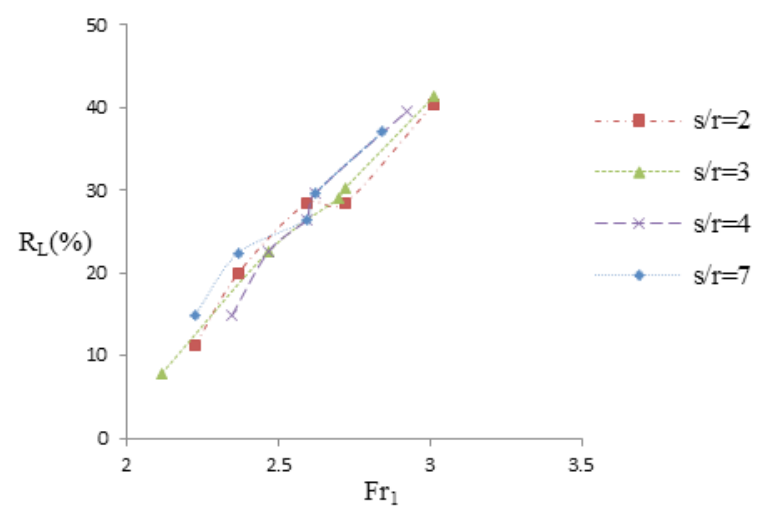

Figure 10. Energy dissipation changes in primary Froude number (2 $\mathrm{cm}$ barriers).

\subsection{The Effect of Barriers Specifications on Determining the Point of Jump}

In hydraulic structures, determining the exact point of hydraulic jump in order to build resting pool and restoring bed bottom in the place jump is formed is very important. Thus in this study the effect of change in relative roughness on the place jump is formed has been investigated. In all vector analyses, the distance of the first barrier from the rectangular overflow claw at the beginning of the canal is constant and only the distance between the barriers has been changed. In order to draw the diagram for determining the point of jump, the factor $\left(\mathrm{L}_{\mathrm{D}} / \mathrm{Y}_{2}\right)$ is defined as length factor in which $\mathrm{L}_{\mathrm{D}}$ is the distance of the beginning of the jump formation from overflow claw and $\mathrm{y}_{2}$ is the secondary depth of the flow. The findings for barriers 2 and $3 \mathrm{~cm}$. are shown in Figures 11 and 12 .

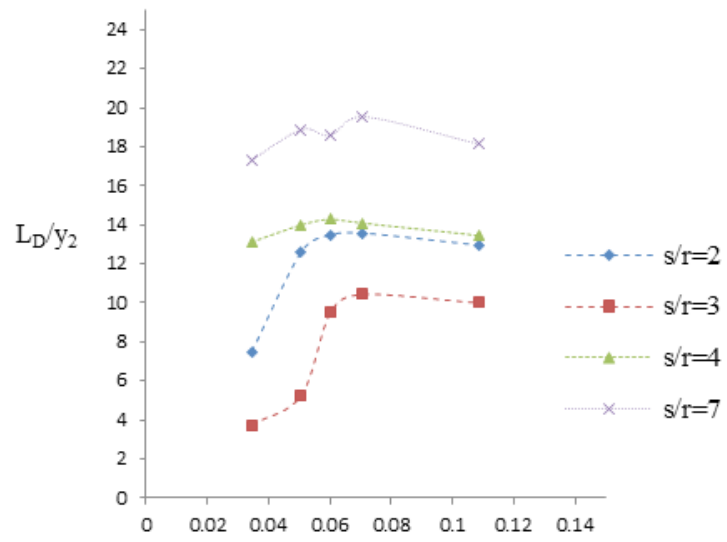

q

Figure 11. The change in the place of jump formation in different discharges of the barriers $(3 \mathrm{~cm})$.

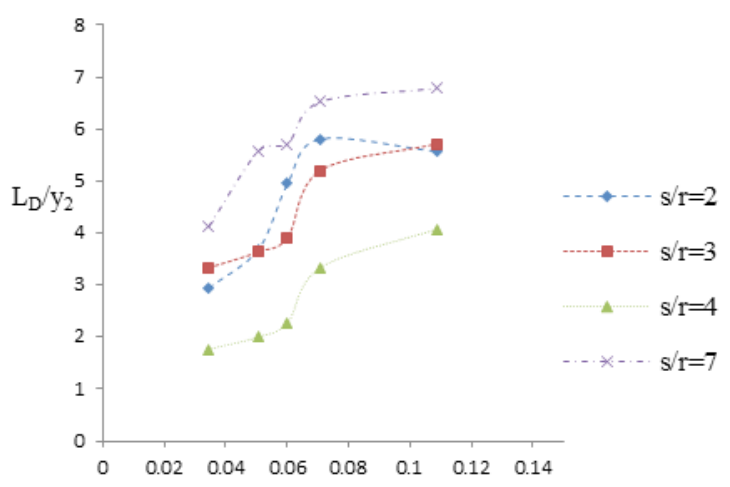

q

Figure 12. The change in the place of jump formation in different discharges of the barriers $(2 \mathrm{~cm})$. 
The findings for $3-\mathrm{cm}$ barrier shows that discharge increase causes increase in the in the distance of jump formation from overflow. The point of jump formation in relative roughness 2 and 3 is almost the same and in relative roughness 4 the jump moves toward overflow and its distance from overflow claw decreases to the minimum. In relative roughness 7 , the distance of jump point of formation from overflow increases and the most distance has been recorded in this state. The findings show that increase in the distance between the barriers and relative roughness 4 cause decreases in length factor and beyond that causes increase in the length factor. The findings for 2-cm barriers also show that the highest length factor is recorded in relative roughness 7 , in this case, the jump has the most distance from overflow claw and in relative roughness 3 and 4 , the point of jump is almost the same and the least length factor for $2-\mathrm{cm}$ barriers is seen in relative roughness 3 . The findings for both diagram indicate that the increase in the distance between the barriers causes increase in the point of jump from the overflow claw at the beginning of the canal.

\section{Conclusion}

By doing 44 tests in the range of Froude 2 to 3.2 a laboratory study was done on the effects of quadrant barriers on forming jump, point of jump formation, energy dissipation and changes in Froude number. Increase in relative roughness factor up to a definite rate cause increase in primary Froude number and beyond that causes decrease in the primary Froude number. for both types of tests the range of relative roughness has been 2 to 4 . The specifications of quadrant barriers including height and the distance between barriers within the desired Froude does affect energy dissipation. The optimal point for increasing energy dissipation for both types of tests has been relative roughness 2 to 4 . Within the desired Froude, change in height and the distance between barriers affects the point of jump in a way that increase in in the distance between barriers up to relative roughness 3 and 4 causes decrease in length factor and beyond that causes increase in the length factor. The amount of optimal relative roughness is in direct relationship with increase in height of the barriers. The more the height of quadrant barriers increases, the more the optimal relative roughness will increase.

\section{References}

1. Pagliara S, Carnacina L, Palermo M. Energy dissipation in presence of block ramps with enlarged stilling basins. 33rd IAHR Congress, Water engineering for asustainable environment; 2009 Aug 4-9; p. 5042-50; Vancouver, Canada

2. Beiramy M, Hosseini AM. Control of hydraulic jump With the continuous wall The horizontal stilling basin. Esteghlal. 2005; 1.

3. Shukry A. The efficiency of floor sills under drowned hydraulic jump. J Hydr Div asce. 1957; 83:1-18,

4. Najandali A, Esmaili K, Farhoudi J. The Effect of Triangular Blocks on the Characteristics of Hydraulic Jump. Journal of Water and Soil. 2012; 26(2):282-9.

5. Abbaspour A, Dalir HA, Farsadizadeh D, Sadraddini AA. Effect of sinusoidal corrugated bed on hydraulic jump characteristics. Journal of Hydro-environment Research. 2009. 3 109e117,

6. Hughes WC, Flack JE. Hydraulic jump properties over a rough bed. Journal of Hydraulic Engineering ASCE. 1984; 110:1755-71.

7. Ead SA, Rajaratnam N. Hydraulic jumps on corrugated beds. Journal of Hydraulic Engineering ASCE. 2002; 128:656-63.

8. Gohari A, Farhoudi J. The characteristics of hydraulic jump on rough bed stilling basins. 33rd IAHR Congress. Water Engineering for a Sustainable Environment; 2009 Aug 9-14; Vancouver, British Columbia.

9. French RH. Hydraulic of open channel flow. Desert Research Institute: University and Community College System of Nevada Reno, Nevada; 2004.

10. Hosseiny M, Abrishami J. Hydraulics of open channels. 28th ed. Publication of Emam Reza University; 2005. 